\title{
Comparison of Serum and Cervical Cytokine Levels throughout Pregnancy between Preterm and Term Births
}

\author{
Kristin Ashford, $\mathrm{PhD}^{1} \quad$ Niraj R. Chavan, MD, MPH $\quad$ Amanda T. Wiggins, $\mathrm{PhD}^{1}$ Molly Malany Sayre, $\mathrm{PhD}^{3}$ \\ Andrea McCubbin, BS ${ }^{1}$ Agatha S. Critchfield, MD $^{4}$ John O’Brien, MD ${ }^{4}$
}

\footnotetext{
${ }^{1}$ Perinatal Research and Wellness Center, University of Kentucky College of Nursing, Lexington, Kentucky

2 Department of Obstetrics \& Gynecology, University of Kentucky College of Medicine, Lexington, Kentucky

${ }^{3}$ Department of Sociology, Anthropology, and Social Work,

University of Dayton, Dayton, Ohio

${ }^{4}$ Division of Maternal-Fetal Medicine, University of Kentucky College of Medicine, Lexington, Kentucky
}

Am J Perinatol Rep 2018;8:e113-e120.

\begin{abstract}
Address for correspondence Molly Malany Sayre, PhD, Department of Sociology, Anthropology, and Social Work, University of Dayton, St. Joseph Hall 401, 300 College Park, Dayton, OH 45469 (e-mail: msayre2@udayton.edu).
\end{abstract}

\begin{abstract}
Keywords

- cytokines

- preterm birth

- serum

- cervicovaginal fluid

Objective To assess differences in cytokine levels in cervicovaginal fluid (CVF) and serum across trimesters between women with preterm births (PTBs) and full-term births.

Study Design This multicenter study enrolled 302 women with a singleton gestation. CVF and serum cytokines, interleukin $1 \alpha$ (IL-1 $\alpha)$, IL-1 $\beta$, IL-6, IL-8, IL-10, C-reactive protein (CRP), tumor necrosis factor (TNF)- $\alpha$, and matrix metalloproteinase (MMP)-8, were measured. Women with at least one cytokine assessment and noted PTB status in their medical record were retained in the study $(N=272)$. Data were analyzed using mixed modeling (main effects of PTBs and time/trimester).

Results For the CVF values of IL-6, IL- 8 , IL-10, TNF- $\alpha$, and CRP, and serum MMP-8, those who delivered preterm had significantly higher values than the full-term group regardless of trimester. For the serum values of IL-1 $\beta$, IL- 6 , and TNF- $\alpha$, those delivering preterm had significantly lower values than those delivering full-term regardless of trimester. For IL-1 $\beta$ in CVF, the cytokine was significantly higher in the PTB group for second and third trimesters only, relative to the full-term group.

Conclusion For each CVF cytokine that differed by birth status, values were higher for PTB than term, averaged over trimester. Numerous cytokine profiles varied across trimesters in women delivering term versus preterm in both CVF and serum.
\end{abstract}

While different cytokines are present in varying concentrations throughout the course of the pregnancy, a balance ensures that the fetus is carried to term. The interleukins (ILs) assessed in this study included IL- $1 \alpha$, IL-1 $\beta$, IL-6, IL-8, and IL-10, while other cytokines, such as tumor necrosis factor (TNF)- $\alpha, C$-reactive protein (CRP), and matrix metalloproteinase (MMP)-8, were also measured. While these cytokines generally play differing roles in the human body, some altera- tions may be an indicator for other adverse pregnancy outcomes, and all cytokines can have adverse effects during pregnancy if their levels are abnormal. IL- $1 \alpha$ and IL-1 $\beta$ peak prior to labor onset, ${ }^{1}$ while IL-6, IL-8, and IL-10 are all components of the inflammatory pathway that must be halted to prevent fetal rejection. ${ }^{2-4}$ CRP is also involved in the inflammatory process during pregnancy and maternal levels can be associated with fetal growth restrictions and increased received

June 12, 2017

accepted after revision

March 24, 2018
DOI https://doi.org/

10.1055/s-0038-1656534. ISSN 2157-6998.
Copyright $\odot 2018$ by Thieme Medical Publishers, Inc., 333 Seventh Avenue, New York, NY 10001, USA. Tel: +1(212) 584-4662.
License terms

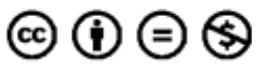


risk of neonatal complications. ${ }^{5}$ TNF- $\alpha$ is a multifunctional cytokine, but its primary role during pregnancy is to terminate development if the embryo has suffered damages that will result in abnormal development. ${ }^{6}$ When this cytokine is released inappropriately, the embryo can be terminated even when lacking abnormalities.

Cytokine concentrations change throughout pregnancy. ${ }^{7}$ Many of those involved with the inflammatory process, including IL- $1 \beta$ and IL-8, decrease to protect the fetus during gestation. ${ }^{8}$ Other lipopolysaccharide-stimulated cytokines, such as IL- 6 and TNF- $\alpha$, have shown a significant increase across the course of pregnancy. ${ }^{8}$ The mechanisms of these changes are not well understood, but the phenomenon has been observed in longitudinal studies. ${ }^{8,9}$ Cytokine levels during pregnancy may be specifically linked to precursors to preterm birth. In particular, the release of inflammatory cytokines, normally in response to a bacterial infection, accounts for approximately half of all preterm births. ${ }^{10,11}$ The objective of this study was to assess whether there are differences in cytokine levels across trimesters between women with preterm and full-term births, controlling for maternal sociodemographic and personal factors. A secondary objective was to contrast findings between cytokine levels obtained from cervicovaginal fluid (CVF) and those obtained from serum.

\section{Materials and Methods}

These data were obtained from a prospective, multicenter trial of women with singleton pregnancies. The longitudinal design assessed demographics and outcomes at each trimester of pregnancy. Potential participants were approached and screened in the first trimester of pregnancy. Exclusion criteria targeted conditions with significant influence on immune markers including preexisting diabetes, heart disease, a medical history of human immunodeficiency virus (HIV), bacterial vaginosis, sexually transmitted infections, any autoimmune disease, or positive drug use. The study was approved by the Institutional Review Boards at each data collection site. Written consent was obtained from all participants, who were given modest compensation at each trimester. Women who completed at least one cytokine assessment were retained in the study $(N=272)$. Of these, there were 257,234 , and 219 with maternal serum measurements and 242, 217, and 194 with CVF measurements across the three trimesters. Cytokines included: IL- $1 \alpha$, IL-1 $\beta$, IL-6, IL-8, IL-10, TNF- $\alpha$, CRP, and MMP-8. Fewer CVF samples were collected, as about $9 \%$ of women did not provide a specimen due to a medical condition (e.g., vaginal bleeding, reports of cramping or discomfort) or refusal. The numbers of samples available for MMP-8 (serum: 185, 164, 150; and CVF: 165, 147,124 , respectively) are lower than other biomarkers, as it was added at study midpoint. Trimester collection windows ranged for 6 weeks $(8-13,18-23$, and 28-33 weeks) with a minimum of 4 weeks between collection periods.

\section{Sample Collection and Cytokine Estimation}

Clotted blood samples were centrifuged at 2,000 rpm for 10 minutes, and the serum was divided into three aliquots and stored at $-80^{\circ} \mathrm{C}$. Samples were analyzed undiluted. For CVF specimens, samples were obtained after the speculum was placed and the cervix visualized. An ectocervical sample was collected by sweeping the cervix 360 degrees and kept in place for 30 seconds to maximize saturation. When removing the swab (source), a vaginal sample was obtained by sweeping 360 degrees in the vaginal vault/posterior fornix. The swab was then placed in the proprietary container, firmly pressed against the inner cryovial wall to ensure maximum seepage of fluid into the buffer container; and the vial cap was secured. All serum and CVF specimens had sufficient amount for analysis, as high-sensitivity assays were used to detect below-minimum concentrations. All samples were immediately refrigerated and were transported to the laboratory within 6 hours, where they were stored at $-20^{\circ} \mathrm{C}$ for a minimum of 24 hours. To further process, samples were thawed at $4^{\circ} \mathrm{C}$ and then centrifuged at $3,750 \mathrm{rpm}$ for 15 minutes. For long-term storage, the samples were split into three aliquots and stored at $-80^{\circ} \mathrm{C}$. Cytokines IL-1 $\alpha$, IL-1 $\beta$, IL-6, IL-8, IL-10, and TNF- $\alpha$ were measured using multiplex Beadlyte assay (MPXHCYTO-60K-06) on a Luminex IS-100 (Austin, TX) according to the manufacturer's recommendations. High-sensitivity testing was used for samples below minimum detectable concentrations. Singleplex assays were used for CRP (Millipore, Billerica, MA) and MMP-8 (R\&D Systems, Minneapolis, MN). The dynamic range of the assays was 50 to $0.016 \mathrm{ng} / \mathrm{mL}$, with a minimum detection concentration of $0.0012 \mathrm{ng} / \mathrm{mL}$. All cytokine data were generated using Milliplex Analyst Software.

\section{Statistical Analysis}

Consistent with prior research, ${ }^{9}$ any CVF or serum cytokine value classified as an outlier(i.e., outside of 3 SD from the mean) was excluded from this analysis. The range in number of excluded values for any given cytokine and trimester was 1 to 11 ; the average number of omitted values across the cytokines and trimesters was $4.4(\mathrm{SD}=1.8)$. Prior to analysis, all cytokine values were log-transformed (natural logarithm) as an adjustment for lack of normality in the raw values. Bivariate analyses, including the two-sample $t$-test or chi-square test of association, were used to compare demographic and personal characteristics between women who experienced preterm versus full-term births. Repeated-measures mixed modeling was used to discern differences in cytokine levels by preterm birth status during pregnancy, using the MIXED procedure in SAS. This method was chosen as it allowed for the inclusion of multiple cytokine values per participant (i.e., one for each trimester they were assessed), and also permitted the inclusion of women who did not have all three assessments in the analysis, whether due to lack of sample, a measure outside the 3 SD limit, or missed assessments due to preterm birth or other reasons.

The primary comparison for each mixed model was between length-of-pregnancy groups (i.e., women with preterm and full-term births). Other factors included in the model were age, race/ethnicity, education, first-trimester smoking status, and trimester of pregnancy. We also included the interaction between length-of-pregnancy group and trimester in the initial models but removed the 
interaction in all but one model (CVF IL-1 $\beta$ ) due to lack of significance of the trimester by group interaction in the full model. This suggests that for all cytokines, with the exception of IL-1 $\beta$ in CVF, the pattern of change over the three trimesters in average cytokine levels did not differ between the two length-of-pregnancy groups. On the other hand, significant main effects of length-of-pregnancy group or trimester for some cytokines suggest that there are differences between the two term groups (averaged over trimester) or among the trimesters (averaged over term group). Post hoc pairwise comparisons for significant main or interaction effects were accomplished using Fisher's least significant difference procedure. All analysis was conducted using SAS v. 9.4 ; an $\alpha$ level of 0.05 was used throughout.

\section{Results}

Of the 272 women with at least one cytokine measurement during at least one trimester who also had their length of pregnancy recorded, 39 had preterm births and the remaining 233 participants had full-term births. No differences in maternal age, body mass index, parity, race, education, income, or smoking status were identified between those who delivered preterm and term (see - Table 1). Among those who delivered preterm, the average gestational age at delivery was 34.3 weeks $(S D=2.7)$. The vast majority of

Table 1 Comparison of characteristics between length-ofpregnancy groups, including all women with at least one cytokine level measured during pregnancy $(N=272)$

\begin{tabular}{|c|c|c|c|}
\hline & $\begin{array}{l}\text { Preterm } \\
(n=39)\end{array}$ & $\begin{array}{l}\text { Full-term } \\
(n=233)\end{array}$ & $p$ \\
\hline $\begin{array}{l}\text { Maternal age, } \\
\text { mean (SD) }\end{array}$ & $26.8(6.4)$ & $26.6(5.1)$ & $0.87^{\mathrm{a}}$ \\
\hline $\begin{array}{l}\text { Body mass index, } \\
\text { mean (SD) }\end{array}$ & $26.6(5.7)$ & $26.5(6.3)$ & $0.98^{\mathrm{a}}$ \\
\hline \multicolumn{4}{|l|}{ Parity, $n(\%)$} \\
\hline 0 & $15(44.1 \%)$ & $123(55.2 \%)$ & \multirow[t]{3}{*}{0.24} \\
\hline 1 & $9(26.5 \%)$ & $47(21.1 \%)$ & \\
\hline 2 or more & $10(29.4 \%)$ & $53(23.8 \%)$ & \\
\hline \multicolumn{4}{|l|}{ Race, $n(\%)$} \\
\hline White & $28(75.7 \%)$ & $176(75.9 \%)$ & \multirow[t]{2}{*}{0.98} \\
\hline Other & $9(24.3 \%)$ & $56(24.1 \%)$ & \\
\hline \multicolumn{4}{|l|}{ Education, $n(\%)$} \\
\hline Less than high school & $3(7.9 \%)$ & $33(14.2 \%)$ & \multirow[t]{2}{*}{0.29} \\
\hline High school or above & $35(92.1 \%)$ & $199(85.8 \%)$ & \\
\hline \multicolumn{4}{|l|}{ Income, $n(\%)$} \\
\hline Less than $\$ 20,000$ & $13(35.1 \%)$ & $63(23.3 \%)$ & \multirow[t]{2}{*}{0.39} \\
\hline$\$ 20,000$ or more & $24(64.9 \%)$ & $160(71.7 \%)$ & \\
\hline \multicolumn{4}{|c|}{ First trimester smoking status, $n(\%)$} \\
\hline Smoker & $12(31.6 \%)$ & $54(24.0 \%)$ & \multirow[t]{2}{*}{0.32} \\
\hline Nonsmoker & $26(68.4 \%)$ & $171(76.0 \%)$ & \\
\hline
\end{tabular}

${ }^{a} G r o u p$ comparison via two-sample $t$-test; all other comparisons via chi-square test of association. deliveries resulted from spontaneous preterm birth $(n=26)$. while others were a result of preterm premature rupture of membranes (PPROM) or iatrogenic.

\section{CVF Levels across Trimesters by Length-of-Pregnancy Status}

Repeated-measures models showed significant main effects of length-of-pregnancy status (i.e., preterm vs. term) averaged over trimester for IL-6, IL-8, IL-10, TNF- $\alpha$, and CRP (see - Table 2), controlling for maternal age, race, education, and first trimester smoking status. For each of the cytokines with significant length-of-pregnancy main effects, the CVF cytokine levels were elevated in those who delivered preterm compared with women with full-term pregnancies (see - Fig. 1). A significant difference in the change of CVF IL-1 $\beta$ levels was noted across pregnancy between women who delivered preterm and term (i.e., significant length-of-pregnancy by trimester interaction effect; see - Fig. 2). Women who delivered preterm had significantly elevated levels at mid and late trimester, compared with those who delivered fullterm; there was no difference in CVF IL- $1 \beta$ between the two groups during early pregnancy (first trimester). No differences were detected in IL- $1 \alpha$ or MMP- 8 by preterm birth status, and the interaction between trimester and length-of-pregnancy was not significant for these two cytokines.

The main effect of trimester, regardless of term status, was significant for the CVF measurements of IL- $1 \alpha$, IL-8, IL-10, TNF- $\alpha$, and MMP- 8 . With the exception of TNF- $\alpha$, each of these cytokines increased as pregnancy progressed. For TNF- $\alpha$, the level decreased during the course of pregnancy (see - Fig. 3 ). For IL- $1 \alpha$, IL-10, and MMP-8, first-trimester measurements were significantly less than the measurements at both second and third trimester, while for IL-8 the first-trimester level was significantly less than only the third trimester. For TNF- $\alpha$, the level at first trimester was significantly greater than both second and third trimesters, while second trimester values were also significantly greater than third.

\section{Serum Levels across Trimesters by Length-of- Pregnancy Status}

Results from the adjusted linear mixed models based on the serum values showed significant main effects of preterm birth status regardless of trimester for IL- $1 \beta$, IL- 6 , TNF- $\alpha$, and MMP-8 (see - Table 2). Serum levels of IL-6, IL-1 1 , and TNF- $\alpha$ were significantly lower for those who delivered preterm relative to full-term deliveries, but MMP-8 was significantly higher (see - Fig. 4). A difference in systemic levels of IL- $1 \alpha$, IL-8, IL-10, or CRP was not observed by preterm birth status. None of the eight cytokines measured exhibited a significant preterm status by trimester interaction.

The main effect of trimester, regardless of preterm/fullterm birth, was significant for serum IL-8, TNF- $\alpha$, and CRP, but not for the other five cytokines assessed. For IL-8, the level at second trimester was significantly less than at third trimester, but the first trimester level did not differ significantly from either of the later levels (see - Fig. 5). For CRP and TNF- $\alpha$, an increase was observed over time; for both cytokines, the first trimester level was significantly less than 
Table 2 Repeated-measures models for each cytokine with main effects of length-of-pregnancy status and trimester

\begin{tabular}{|c|c|c|c|c|c|c|}
\hline & \multicolumn{3}{|l|}{ CVF } & \multirow[b]{2}{*}{$n$} & \multicolumn{2}{|c|}{ Serum } \\
\hline & $n$ & $F$ & $p$ & & $F$ & $p$ \\
\hline \multicolumn{7}{|l|}{ IL-1 $\alpha$} \\
\hline Length-of-pregnancy & \multirow[t]{2}{*}{226} & 0.2 & 0.64 & \multirow[t]{2}{*}{243} & 3.7 & 0.054 \\
\hline Trimester & & 4.6 & 0.010 & & 1.9 & 0.15 \\
\hline \multicolumn{7}{|l|}{ IL-1 } \\
\hline Length-of-pregnancy & \multirow[t]{3}{*}{223} & 11.4 & $<0.001$ & \multirow[t]{3}{*}{240} & 4.9 & 0.028 \\
\hline Trimester & & 4.6 & 0.011 & & $<0.1$ & 0.99 \\
\hline Length-of-pregnancy $\times$ trimester & & 3.0 & 0.049 & & - & - \\
\hline \multicolumn{7}{|l|}{ IL-6 } \\
\hline Length-of-pregnancy & \multirow[t]{2}{*}{224} & 7.3 & 0.007 & \multirow[t]{2}{*}{242} & 9.1 & 0.0028 \\
\hline Trimester & & 0.6 & 0.53 & & 0.4 & 0.69 \\
\hline \multicolumn{7}{|l|}{ IL-8 } \\
\hline Length-of-pregnancy & \multirow[t]{2}{*}{227} & 4.2 & 0.042 & \multirow[t]{2}{*}{243} & 1.0 & 0.32 \\
\hline Trimester & & 3.1 & 0.046 & & 3.0 & 0.049 \\
\hline \multicolumn{7}{|l|}{ IL-10 } \\
\hline Length-of-pregnancy & \multirow[t]{2}{*}{225} & 20.8 & $<0.001$ & \multirow[t]{2}{*}{239} & 0.5 & 0.49 \\
\hline Trimester & & 16.4 & $<0.001$ & & 1.8 & 0.16 \\
\hline \multicolumn{7}{|l|}{ CRP } \\
\hline Length-of-pregnancy & \multirow[t]{2}{*}{227} & 7.2 & 0.0079 & \multirow[t]{2}{*}{234} & 1.2 & 0.27 \\
\hline Trimester & & 2.2 & 0.11 & & 4.4 & 0.013 \\
\hline \multicolumn{7}{|l|}{ TNF- $\alpha$} \\
\hline Length-of-pregnancy & \multirow[t]{2}{*}{226} & 26.5 & $<0.001$ & \multirow[t]{2}{*}{240} & 43.1 & $<0.001$ \\
\hline Trimester & & 10.1 & $<0.001$ & & 9.1 & $<0.001$ \\
\hline \multicolumn{7}{|l|}{ MMP-8 } \\
\hline Length-of-pregnancy & \multirow[t]{2}{*}{158} & 0.2 & 0.62 & \multirow[t]{2}{*}{180} & 5.4 & 0.022 \\
\hline Trimester & & 3.7 & 0.027 & & 2.9 & 0.056 \\
\hline
\end{tabular}

Abbreviations: CRP, C-reactive protein; CVF, cervicovaginal fluid; IL, interleukin; MMP, matrix metalloproteinase; TNF, tumor necrosis factor. Note: Models adjusted for age, race/ethnicity, education, and first trimester smoking status; only those with complete data on all covariates, and those with cytokine data at any trimester, are included in the model.

(a) IL-6 $(n=244)$

(a) $-1(n=244)$

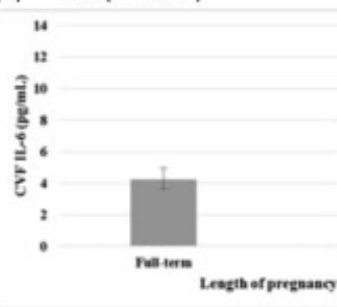

(d) TNF- $\alpha(n=226)$

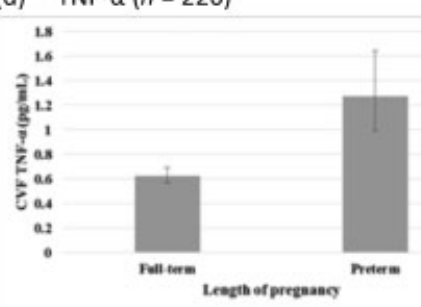

(b) $\mathrm{IL}-10(n=225)$

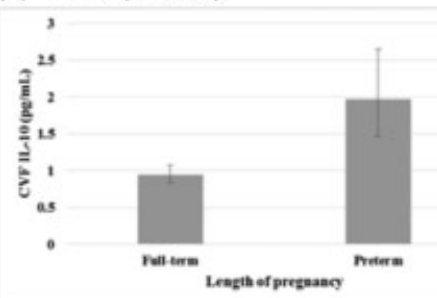

(c) $\operatorname{CRP}(n=227)$

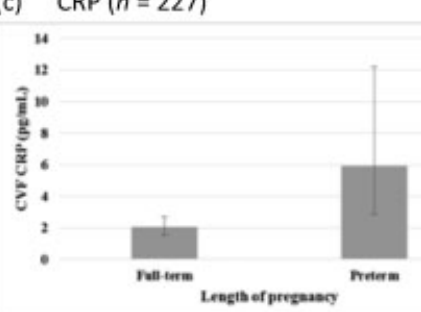

Fig. 1 Mean (95\% confidence interval $[\mathrm{Cl}])$ untransformed CVF levels among women during pregnancy significantly varying by length of pregnancy. (a) IL-6, (b) IL-10, (c) CRP, (d) TNF- $\alpha$. 


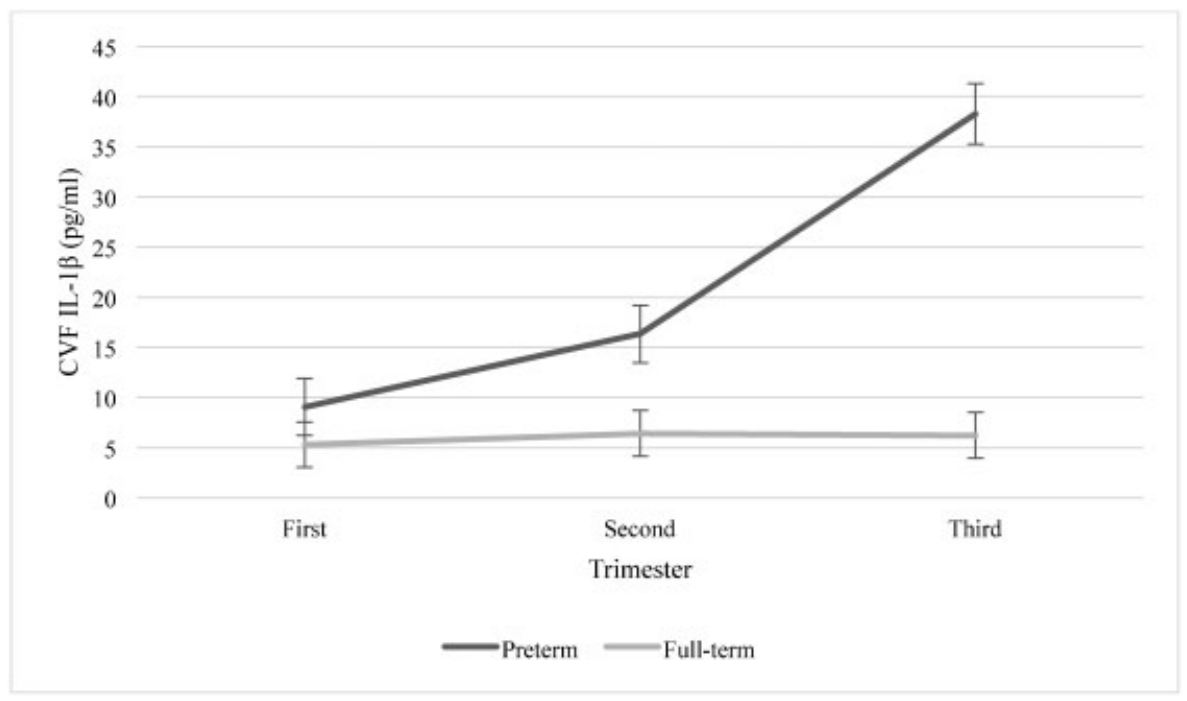

Fig. 2 Mean $(95 \% \mathrm{Cl})$ untransformed CVF IL-1 $\beta$ levels among women during pregnancy by preterm birth status at each trimester. Repeatedmeasures models showed significant elevated levels among women who delivered preterm in both second $(t=2.2 ; p=0.029)$ and third trimesters $(t=3.9 ; p<0.001)$.
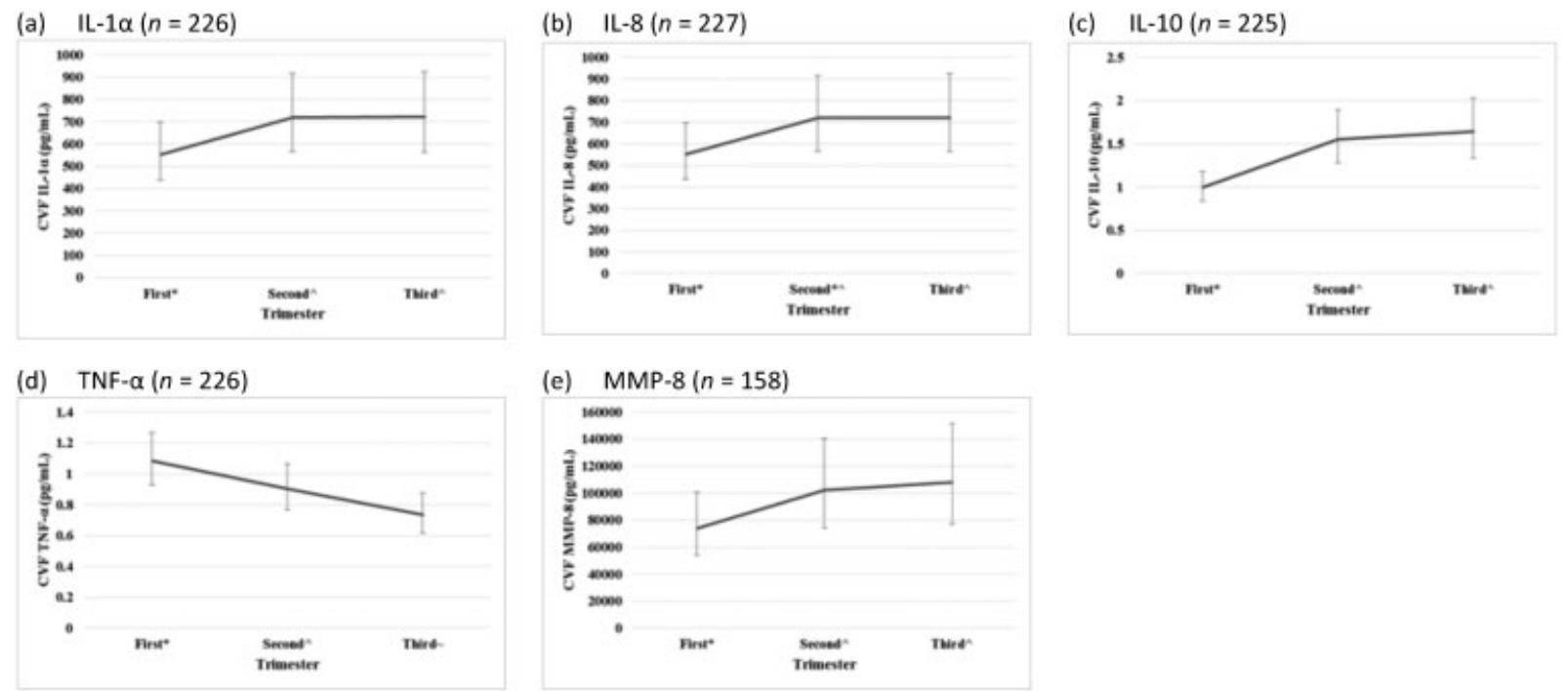

Fig. 3 Mean $(95 \% \mathrm{Cl})$ untransformed CVF levels among women significantly varying during pregnancy by trimester. (a) IL-1 $\alpha$, (b) IL-8, (c) IL-10, (d) TNF- $\alpha,(e)$ MMP-8.

both second and third, while for TNF- $\alpha$ the second trimester level was also significantly less than that of the third trimester.

\section{Discussion}

For each CVF cytokine that differed by length-of-pregnancy status, values were higher for preterm than full-term births, averaged over trimester. This included significant differences in IL-6, IL-8, IL-10, TNF- $\alpha$, and CRP between women who delivered preterm versus full-term. This is consistent with previous research findings on higher levels of CVF IL-8 associated with gestational age, ${ }^{12}$ spontaneous preterm birth, ${ }^{13}$ and, among women with preterm labor and without PPROM, delivery within 7 days. ${ }^{14}$ One study found no asso- ciation between CVF IL-8 and cervical shortening at 20 or 24 weeks' gestation; ${ }^{15}$ given the difference between this result and our present findings, changes in IL- 8 may be better detected later in pregnancy, or may be associated with other symptoms of preterm birth.

A strength of this study is that it is one of the first to examine cytokines in both serum and CVF across all three trimesters of pregnancy. Also, studies examining preterm birth often do not validate smoking status, often due to feasibility or cost. Our study is also among the first to use biochemical validation of smoking status (via urine cotinine) at each time point.

Several studies have found associations between CVF IL-6 and preterm birth. Higher levels of CVF IL- 6 were associated with delivery within 7 days among women with preterm 
(a) IL-6 ( $n=242)$

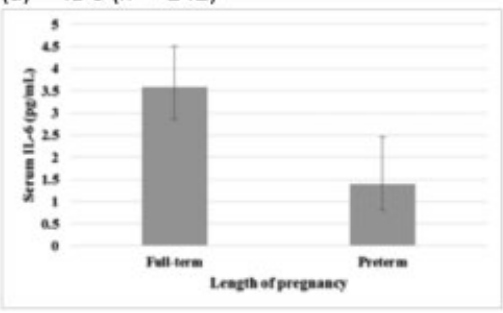

(b) TNF- $\alpha(n=240)$

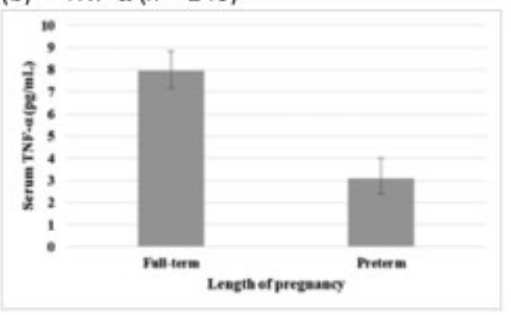

(c) $\operatorname{MMP}-8(n=180)$

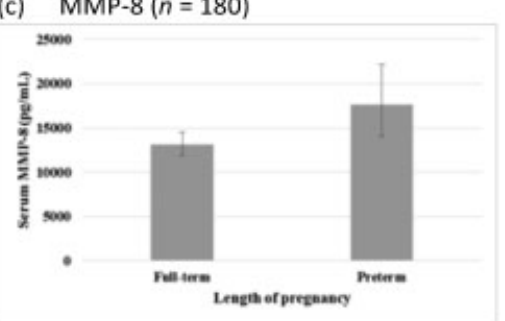

Fig. 4 Mean $(95 \% \mathrm{Cl})$ untransformed serum levels among women during pregnancy significantly varying by length of pregnancy. (a) IL-6, (b) TNF- $\alpha$, (c) MMP-8.
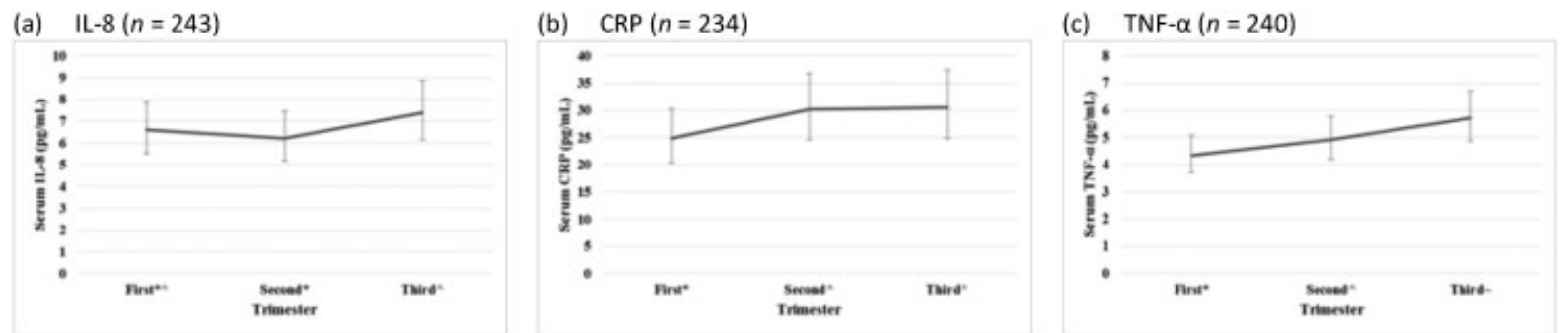

Fig. 5 Mean $(95 \% \mathrm{Cl})$ untransformed serum levels among women during pregnancy significantly varying by trimester. (a) IL-8, (b) CRP, (c) TNF- $\alpha$.

labor. ${ }^{14}$ In a sample of women tested at hospital admission, CVF IL-6 was significantly higher among women with PPROM compared with controls, matched for gestational age. ${ }^{16}$ Like our investigation, Reyna-Vallasmil et al found CVF IL-6, measured in the second trimester, was significantly higher among women who later delivered preterm. ${ }^{17}$ In contrast, a study by Cox et al with full-term pregnancies did not demonstrate CVF IL-6 to be significantly different between women either in labor or not in labor. ${ }^{18}$

In congruence with our findings, CVF CRP was found to be associated with microbial invasion of the amniotic cavity (MIAC) in women with PPROM. ${ }^{19}$ Our findings, however, are in contrast to those of Cobo et al, who found that CVF TNF- $\alpha$ was undetectable in over $50 \%$ of a sample of women with PPROM, ${ }^{19}$ as well as Seong et al, who found no association between TNF- $\alpha$ and cervical shortening at 20 or 24 weeks' gestation. $^{15}$

Dutt et al found that CVF IL-10 was significantly higher in non-Caucasian than Caucasian women in both the second and third trimesters of pregnancy. ${ }^{20}$ In fact, the median CVF IL-10 levels among Caucasian women were below the level of detection in each trimester, so it is notable that IL-10 was associated with preterm birth in the present study, conducted with a mostly Caucasian (75.8\%) sample. It is possible that Dutt et al's non-Caucasian sample and the present study's sample share salient similarities (e.g., lower-thanaverage household income, cumulative life stress) which contribute to higher levels of CVF IL-10 and an association with preterm birth.

The findings of elevated CVF cytokines, averaged over trimester, among women who delivered preterm are in contrast with serum cytokine values: for these, nearly all of the cytokines that exhibited a significant difference for the term/ preterm comparison were lower for preterm births compared with term. This is true for IL-1 $\beta$, IL-6, and TNF- $\alpha$, when averaged over trimester. This finding is very intriguing and a value of the study. Our finding suggests that expression of the inflammatory response is not consistent across all biologic pools and, for pregnancy, appears concentrated on the local cervical reaction when a pathophysiology potentially responsible for preterm birth is present. Only MMP-8 measured in serum was higher for preterm than term deliveries. A composite profile of inflammatory mediators may help improve the prediction for preterm birth and the extent of the response to an ongoing pathophysiology.

Others have found differences in CVF versus serum cytokine levels relative to preterm birth. Yavari Kia et al found significant differences and no correlation between serum and CVF levels of IL-6 among women with preterm uterine contractions. ${ }^{21}$ Since elevated inflammatory biomarkers are thought to indicate higher risk of earlier birth, our findings concur with Yavari Kia et al's conclusion that testing for serum cytokine levels would be not be clinically useful for predicting preterm birth.

Gillespie et al found that IL-1 $\beta$, IL-6, and TNF- $\alpha$ increased from early to late pregnancy among a sample of women with a mean gestational age at delivery of over 39 weeks (SD $=1.0-1.1) \cdot{ }^{22}$ Other studies, including one by Ferguson et al, have found a similar trajectory as Gillespie et al for IL-6 (increasing from 20 weeks' gestation to the end of pregnancy). ${ }^{23,24}$ However, mean values of serum IL-6 were significantly higher for women who delivered preterm than full-term in Ferguson et al's sample, and there was no significant difference in serum IL-1 $\beta$ or TNF- $\alpha$ between length-of-pregnancy groups. Our finding that serum IL-1 $\beta$, IL-6, and TNF- $\alpha$ levels were lower, when averaged over trimester, for women with preterm births than full-term births may suggest that the women who delivered preterm 
had not yet reached peak levels of these serum cytokines. Alternatively, it is possible that the women who delivered preterm may have a different pattern or trajectory of serum cytokines than Gillespie et al's sample of women, most of whom delivered full-term, and Ferguson et al's sample, which had a higher proportion of nonsmokers.

There was a significant interaction between preterm status and trimester for IL-1 $\beta$ only. Women who delivered preterm had significantly higher CVF IL- $1 \beta$ values at second and third trimester, compared with full-term births, but there was no difference between the preterm status groups during first trimester in CVF IL-1 $\beta$. One study found associations between CVF IL-1 $\beta$ and gestational age, active labor, and, among women with full-term pregnancies, cervical dilation. $^{12}$ Other studies, however, found IL-1 $\beta$ to be not associated with cervical length in the second trimester, ${ }^{15}$ nor to be an independent predictor of MIAC. ${ }^{19}$ While CVF IL-1 $\beta$ may not be associated with specific symptoms of preterm birth (cervical shortening, MIAC), our findings indicate a unique difference in CVF IL-1 $\beta$ between women with preterm and full-term deliveries.

For the main effect of trimester (averaging term/preterm groups together), there tended to be an increase in level over time for CVF cytokine values. This is true for and consistent with other studies on IL- $1 \alpha$, IL- $8,{ }^{12}$ IL-10, and MMP-8. CVF IL-10 also increased over time, which is similar to Dutt et al's finding for non-Caucasian, but not for Caucasian, women. ${ }^{20}$ However, CVF TNF- $\alpha$ had the opposite relationship: a decrease over time. This is potentially congruent with earlier findings that CVF TNF- $\alpha$ was undetectable for over $50 \%$ of a sample of women with PPROM before 34 weeks' gestation. ${ }^{19}$ Taken together, it is possible that TNF- $\alpha$ levels decreased during pregnancy to the point of going below the threshold of detection for pregnancies further complicated by PPROM.

The serum values of IL- 8 , TNF- $\alpha$, and CRP had similar patterns over time, with a tendency toward increase in level over trimester. Earlier studies have found similar trajectories for serum TNF- $\alpha,{ }^{22,23}$ but Ferguson et al found a quadratic trajectory for serum CRP: increasing until 20 weeks' gestation, and then decreasing until delivery. ${ }^{23}$ Our findings also differ from another study's result of no significant difference in serum IL-8 from first to third trimester, though this was found in a much smaller sample $(n=26){ }^{24}$

\section{Limitations}

The study's limitations include the 6-week data collection windows in each trimester, which may not account for individual differences in cytokine expression within the same 6-week period. Another limitation was attrition that occurred throughout the trimesters.

\section{Conclusion}

This analysis found differences in cytokine levels throughout pregnancy between women with preterm and full-term births. For each CVF cytokine that differed by birth status, values were higher for preterm than term births, averaged over trimester. Numerous cytokine profiles varied across trimesters in women delivering term versus preterm in both CVF and serum. These differences indicate the need for further research on connections between maternal cytokine profiles and birth outcomes.

\section{Conflict of Interest}

Dr. Ashford reports grants from NIH/NCRR Center for the Biologic Basis of Oral/Systemic Diseases during the conduct of the study.

\section{Acknowledgments}

Research funding was provided by two grants awarded to Kristin Ashford from the National Institutes for Health: Building Interdisciplinary Research Careers in Women's Health (BIRCWH: K12DA14040) and Center for Biomedical Research Excellence (COBRE: 5P20GM103538). This research was also funded in part by the University of Kentucky Clinical and Translational Research Center KL2RR033171 (CTSA grant number NIH CTSA UL1TR000117).

\section{References}

1 Heng YJ, Liong S, Permezel M, Rice GE, Di Quinzio MK, Georgiou HM. The interplay of the interleukin 1 system in pregnancy and labor. Reprod Sci 2014;21(01):122-130

2 Prins JR, Gomez-Lopez N, Robertson SA. Interleukin-6 in pregnancy and gestational disorders. J Reprod Immunol 2012;95 $(1-2): 1-14$

3 Shimoya K, Matsuzaki N, Taniguchi T, et al. Human placenta constitutively produces interleukin-8 during pregnancy and enhances its production in intrauterine infection. Biol Reprod 1992;47(02):220-226

4 Thaxton JE, Sharma S. Interleukin-10: a multi-faceted agent of pregnancy. Am J Reprod Immunol 2010;63(06):482-491

5 Ernst GDS, de Jonge LL, Hofman A, et al. C-reactive protein levels in early pregnancy, fetal growth patterns, and the risk for neonatal complications: the Generation R Study. Am J Obstet Gynecol 2011;205(02):132.e1-132.e12

6 Toder V, Fein A, Carp H, Torchinsky A. TNF- $\alpha$ in pregnancy loss and embryo maldevelopment: a mediator of detrimental stimuli or a protector of the fetoplacental unit? J Assist Reprod Genet 2003;20 (02):73-81

7 Holtan SG, Chen Y, Kaimal R, et al. Growth modeling of the maternal cytokine milieu throughout normal pregnancy: macrophage-derived chemokine decreases as inflammation/counterregulation increases. J Immunol Res 2015;2015:952571

8 Christian LM, Porter K. Longitudinal changes in serum proinflammatory markers across pregnancy and postpartum: effects of maternal body mass index. Cytokine 2014;70(02):134-140

9 Mitchell AM, Palettas M, Christian LM. Fetal sex is associated with maternal stimulated cytokine production, but not serum cytokine levels, in human pregnancy. Brain Behav Immun 2017; 60:32-37

10 Mor G, Cardenas I, Abrahams V, Guller S. Inflammation and pregnancy: the role of the immune system at the implantation site. Ann N Y Acad Sci 2011;1221:80-87

11 Mor G, Cardenas I. The immune system in pregnancy: a unique complexity. Am J Reprod Immunol 2010;63(06):425-433

12 Tanaka Y, Narahara H, Takai N, Yoshimatsu J, Anai T, Miyakawa I. Interleukin-1beta and interleukin-8 in cervicovaginal fluid during pregnancy. Am J Obstet Gynecol 1998;179(3, Pt 1):644-649 
13 Raiche E, Ouellet A, Berthiaume M, Rousseau É, Pasquier JC. Short and inflamed cervix predicts spontaneous preterm birth (COLIBRI study). J Matern Fetal Neonatal Med 2014;27(10):1015-1019

14 Jung EY, Park JW, Ryu A, Lee SY, Cho SH, Park KH. Prediction of impending preterm delivery based on sonographic cervical length and different cytokine levels in cervicovaginal fluid in preterm labor. J Obstet Gynaecol Res 2016;42(02):158-165

15 Seong WJ, Lee DY, Koo TB. Do the levels of tumor makers or proinflammatory cytokines in mid-trimester cervical fluid predict early-stage cervical shortening? J Obstet Gynaecol Res 2015; 41(11):1715-1720

16 Beneventi F, Locatelli E, De Amici M, et al. Soluble HLA-G concentrations in maternal blood and cervical vaginal fluid of pregnant women with preterm premature rupture of membranes. J Reprod Immunol 2016;116(116):76-80

17 Reyna-Vallasmil E, Mejia-Montilla J, Reyna-Villasmil N, et al. Cervicovaginal interleukin- 6 in the prediction of preterm delivery. Rev Peru Ginecol Obstet 2016;62(02):175-181

18 Cox SM, King MR, Casey ML, MacDonald PC. Interleukin- 1 beta, -1 alpha, and -6 and prostaglandins in vaginal/cervical fluids of pregnant women before and during labor. J Clin Endocrinol Metab 1993;77(03):805-815
19 Cobo T, Jacobsson B, Kacerovsky M, et al. Systemic and local inflammatory response in women with preterm prelabor rupture of membranes. PLoS One 2014;9(01):e85277

20 Dutt R, Raker C, Anderson BL. Ethnic variations in cervical cytokine concentrations and vaginal flora during pregnancy. Am J Reprod Immunol 2015;73(02):141-150

21 Yavari Kia P, Baradaran B, Shahnazi M, Asghari Jafarabadi M, Khaze V, Pourasad Shahrak S. Maternal serum and cervicovaginal IL-6 in patients with symptoms of preterm labor. Iran J Immunol 2016; 13(03):229-236

22 Gillespie SL, Porter K, Christian LM. Adaptation of the inflammatory immune response across pregnancy and postpartum in Black and White women. J Reprod Immunol 2016;114: 27-31

23 Ferguson KK, McElrath TF, Chen YH, Mukherjee B, Meeker JD. Longitudinal profiling of inflammatory cytokines and C-reactive protein during uncomplicated and preterm pregnancy. Am J Reprod Immunol 2014;72(03):326-336

24 Hebisch G, Neumaier-Wagner PM, Huch R, von Mandach U. Maternal serum interleukin- 1 beta, -6 and -8 levels and potential determinants in pregnancy and peripartum. J Perinat Med 2004; 32(06):475-480 